\title{
Miniaturized Broadband ENG ZOR Antenna Using a High Permeability Substrate
}

\author{
Seung-Tae Ko $\cdot$ Jeong-Hae Lee
}

\begin{abstract}
This paper presents a miniaturized epsilon negative (ENG) zeroth-order resonance (ZOR) patch antenna with an improved bandwidth. The miniaturization and the broad bandwidth of the ENG ZOR patch antenna are achieved by using a meandered via and a high permeability substrate instead of a straight via and a dielectric substrate. The use of a meandered via allows miniaturization of the ENG ZOR patch antenna without narrowing the bandwidth. The use of a high permeability substrate allows further miniaturization of the ENG ZOR patch antenna and improvement of the bandwidth. A high permeability substrate consisting of a multi-layered substrate is designed to have a small material loss. The antenna $(k r=0.32)$ has a $10 \mathrm{~dB}$ fractional bandwidth of $\sim 1 \%$, which is 1.74 times as broad as that of an antenna with a dielectric substrate.
\end{abstract}

Key words: Epsilon Negative (ENG), Zeroth-Order Resonance (ZOR), Meandered Via, High Permeability Substrate, Multi-Layered Substrate.

\section{Introduction}

Many efforts have been made recently to develop miniaturized antennas having broad bandwidth for use in wireless communication systems. For instance, the epsilon negative (ENG) zeroth-order resonance (ZOR) mushroom patch antenna, which is a low profile omnidrectional radiator, was proposed to achieve miniaturization of a patch antenna [1]. Many ENG ZOR patch antennas are designed by using a high permittivity $\left(\varepsilon_{r}>1\right)$ substrate or a periodic structure [1], [2]. These antennas can be further miniaturized, but they suffer from narrow bandwidth.

In this paper, we investigate two methods for designing a miniaturized ENG ZOR patch antenna with broad bandwidth. First, when a straight via of the conventional mushroom ENG ZOR patch antenna is replaced by a meandered via, this induces a large shunt inductance. Using the meandered via, the miniaturization of an antenna can be achieved without narrowing the bandwidth [3]. Second, a high permeability substrate $\left(\mu_{r}>1\right)$ is utilized instead of a high permittivity substrate. A high permeability substrate is well known to allow a patch antenna to have broad bandwidth [4] [6]. As a high permeability substrate, we use a multi-layered substrate (consisting of dielectric and ferrite layers) that is designed to have a small material loss. The miniaturized ENG ZOR patch antennas are designed by combining a meandered

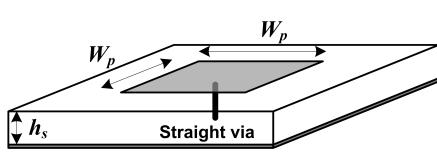

(a) Structure of a conventional mushroom ENG ZOR patch antenna

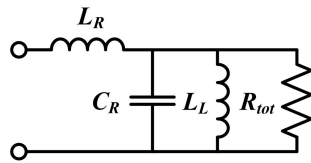

(b) Equivalent circuit $\left(R_{\text {tot }}=R_{\text {rad }}+R_{\text {loss }}\right)$

Fig. 1. Conventional mushroom ENG ZOR patch antenna and its equivalent circuit.

via and a high permeability substrate. Finally, the theoretical and experimental results are compared.

\section{II . The ENG ZOR Patch Antenna with a Meandered Via}

First, a straight via is replaced by a meandered via to obtain a broad bandwidth. The structure of the conventional mushroom ENG ZOR patch antenna and its equivalent circuit are shown in Fig. 1. The mushroom structure is typically composed of a patch and a straight via. Thus, the corresponding shunt inductance $\left(L_{L}\right)$ and capacitance $\left(C_{R}\right)$ are induced by a via and a patch, respectively. The ZOR frequency is then obtained by the open-ended boundary condition, and is given by [7]:

$$
f_{\text {ZOR }}=\frac{1}{2 \pi \sqrt{L_{L} C_{R}}}
$$

Manuscript received April 1, 2011 ; revised July 1, 2011. (ID No. 20110401-016J)

Department of Electronic and Electrical Engineering, Hongik University, Seoul, Korea.

Corresponding Author : Jeong-Hae Lee (e-mail : jeonglee@hongik.ac.kr) 
The shunt capacitance $\left(C_{R}\right)$ is calculated as:

$$
C_{R}=\varepsilon_{0} \varepsilon_{r} \frac{W_{p}^{2}}{h_{S}} .
$$

In this equation, a high permittivity substrate causes a large shunt capacitance $\left(C_{R}\right)$. The $Q$ factor of a parallel resonance circuit is written by [8]:

$$
Q=\frac{R_{t o t}}{\omega_{0} L_{L}}=\omega_{0} R_{t o t} C_{R}
$$

where $R_{\text {tot }}$ includes the radiation loss $\left(R_{r a d}\right)$ and the material loss $\left(R_{\text {loss }}\right)$. Equation (3) indicates that a large shunt inductance $\left(L_{L}\right)$ and a small shunt capacitance $\left(C_{R}\right)$ are required in order to have a broad bandwidth.

Fig. 2 shows two structures of the ENG ZOR patch antennas. The conventional mushroom ENG ZOR patch antenna is shown in Fig. 2(a). This antenna is based on a dielectric substrate that has a relative permittivity of 2.6. The antenna in Fig. 2(b) is the ENG ZOR patch antenna with a meandered via that is placed in the edge of the patch. The antenna in Fig. 2(b) is based on an air substrate. Both antennas are the same size, with the following dimensions: patch width $(W)$ of $150 \mathrm{~mm}(0.1$ $\left.\lambda_{0}\right)$, substrate width $\left(W_{s}\right)$ of $250 \mathrm{~mm}\left(0.166 \lambda_{0}\right)$, and height $\left(h_{s}\right)$ of $30 \mathrm{~mm}\left(0.02 \lambda_{0}\right)$. Both antennas are also designed to have the same operating frequency of 200 $\mathrm{MHz}$. In order to obtain the same operating frequency, a proper dielectric substrate is applied to the antenna in Fig. 2(a).

Fig. 3 shows the simulated return losses for the two antennas using the full wave simulator (Ansoft HFSS). The intrinsic input impedance of the antenna without other external influences is obtained by directly connecting a port between the patch and ground. The input impedance can then be obtained from the simulation result. When the input impedance is normalized as a peak value, a fully matched $S_{11}$ is obtained.

The simulated results for lossless case are shown in Fig. 3(a). The antenna in Fig. 2(a) has a resonance frequency of $194.9 \mathrm{MHz}$ and a $10 \mathrm{~dB}$ fractional bandwidth

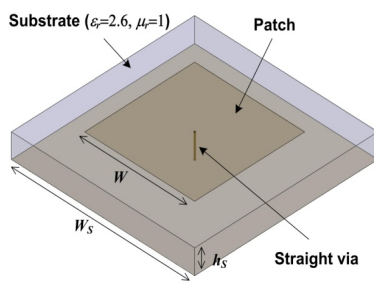

(a) Conventional mushroom ENG ZOR patch antenna

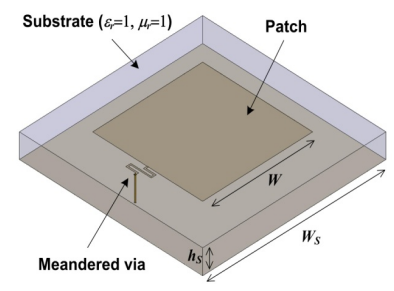

(b) ENG ZOR patch antenna with a meandered via
Fig. 2. One cell ENG ZOR patch antennas.

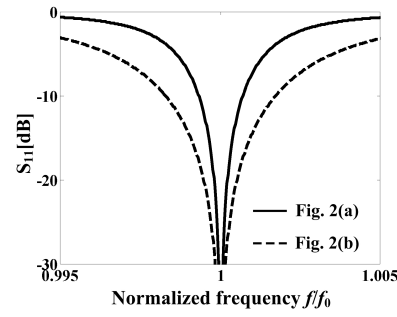

(a) The lossless case

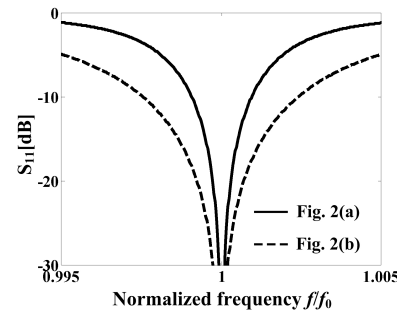

(b) The lossy case
Fig. 3. Simulated return losses.

of $0.14 \%$. The antenna in Fig. 2(b) has a resonance frequency of $196.5 \mathrm{MHz}$ and a broader $10 \mathrm{~dB}$ bandwidth of $0.35 \%$. The radiation efficiencies of the two antennas are obtained at $100 \%$ because no loss is considered. The simulated results, including a conductor loss ( $\sigma$ $\left.{ }_{c}=5.8 \times 10^{7} \mathrm{~S} / \mathrm{m}\right)$, are shown in Fig. 3(b). The figure shows that each resonance frequency is unchanged, but the radiation efficiencies are decreased because the conductor loss is considered. Thus, the $10 \mathrm{~dB}$ fractional bandwidth is broader than for the lossless case. The antennas in Fig. 2(a) and (b) have the $10 \mathrm{~dB}$ bandwidth of $0.19 \%$ and $0.49 \%$, respectively. The radiation efficiencies of the two antennas that include a conductor loss are also obtained as $73.3 \%$ and $70.7 \%$, respectively. The antenna miniaturization can be achieved by using a high permittivity substrate, but its bandwidth becomes too narrow because of the induction of the large shunt capacitance by a high permittivity substrate, as shown for the antenna in Fig. 2(a). However, the antenna in Fig. 2(b) has a broader bandwidth than the antenna in Fig. 2(a), even though the size and the resonance frequency are equal. The meandered via allows an ENG ZOR patch antenna to have a large inductance, resulting in broader bandwidth.

\section{The ENG ZOR Patch Antenna with a High Permeability Substrate}

Second, a broad bandwidth is also obtained by using a high permeability substrate instead of a high permittivity substrate. In general, the resonance frequency of the conventional patch antenna is known to depend on $\varepsilon_{r} \mu_{r}$. Therefore, the value of relative permeability $\left(\mu_{r}\right)$ is the same as that of relative permittivity $\left(\varepsilon_{r}\right)$ for antenna miniaturization. The $10 \mathrm{~dB}$ fractional bandwidth for a dominant resonance mode of the conventional patch antenna is given by [9]:

$$
\text { Fractional } B W[\%] \approx \sqrt{\frac{\mu_{r}}{\varepsilon_{r}}} \frac{9600 h_{S}}{\sqrt{2} \lambda_{0}\left[4+17 \sqrt{\mu_{r} \varepsilon_{r}}\right]}
$$

where $h_{s}$ is a height of the substrate. According to (4), 
Table 1. Simulated bandwidth of the ENG, ZOR antenna in Fig. 2(b) with various substrates.

\begin{tabular}{|c|c|c|c|}
\hline$\left(\varepsilon_{r}, \mu_{r}\right)$ & $(25,1)$ & $(5,5)$ & $(1,25)$ \\
\hline $\begin{array}{c}10 \mathrm{~dB} \text { fractional } \\
\text { BW }(\%)\end{array}$ & $9.0 \times 10^{-4}$ & $4.0 \times 10^{-3}$ & $8.0 \times 10^{-3}$ \\
\hline
\end{tabular}

the miniaturized ENG ZOR patch antenna having a broad bandwidth can be designed when a high permittivity substrate is replaced by a high permeability substrate.

Table1 shows the simulated bandwidths for the ENG ZOR patch antenna in Fig. 2(b), with three different substrates, while $\varepsilon_{r} \mu_{r}$ is fixed to keep the same size of antenna. Table 1 shows that the $10 \mathrm{~dB}$ fractional bandwidths are obtained as $9.0 \times 10^{-4} \%, 4.0 \times 10^{-3} \%$ and $8.0 \times 10^{-3} \%$ when the values of relative permittivity and relative permeability of the substrate are given by $\left(\varepsilon_{r}=\right.$ 25, $\left.\mu_{r}=1\right),\left(\varepsilon_{r}=5, \mu_{r}=5\right)$ and $\left(\varepsilon_{r}=1, \mu_{r}=25\right)$, respectively. Note that no loss is included in this simulation. As expected, a high permeability substrate allows the antenna to have the broadest bandwidth.

\section{The Multi-layered Substrate with High Permeability}

Since most ferrites have hundreds or even thousands of permeability values, a high dielectric (or magnetic) loss, and a dispersive property, they are difficult to apply to ENG ZOR antennas. In this paper, we used a Co2Z-type $\left(\mathrm{Ba}_{3} \mathrm{Co}_{2} \mathrm{Fe}_{24} \mathrm{O}_{41}\right)$ hexaferrite material with a relative permittivity of 12 , a relative permeability of 10 , and a magnetic loss tangent $\left(\tan \delta_{m}\right)$ of 0.03 around 200 MHz. This hexaferrite is manufactured by Trans-Tech Inc. [10]. Although the hexaferrite has a relatively large permeability, it still has several problems. Some modifications are required in order to obtain a smaller relative permittivity and a lower $\tan \delta_{m}$. Thus, we used the multi-layered substrate shown in Fig. 4. The structure of Fig. 4 consists of 2 layers of hexaferrite and 3 layers of RT/duroid5880 substrates. The RT/duroid5880 substrate has a relative permittivity of 2.2 , a relative permeability of 1 , and a dielectric loss tangent $\left(\tan \delta_{d}\right)$ of $9.0 \times 10^{-4}$. The heights of the RT/duroid5880 and the hexaferrite substrates are $9.51 \mathrm{~mm}$ and $2.5 \mathrm{~mm}$, respectively. Thus, the total height of the multi-layered substrate is 33.53 $\mathrm{mm}$.

To confirm the characteristics of the multi-layered substrate, the equivalent relative permittivity and the equivalent relative permeability need to be extracted. Since the RT/duroid5880 and hexaferrite substrates have material losses, the equivalent parameters should be a complex number. Note that the equivalent permittivity and the equivalent permeability can be expressed as $\varepsilon_{e q}=\varepsilon^{\prime}-j$ $\varepsilon^{\prime \prime}$ and $\mu_{e q}=\mu^{\prime}-j \mu^{\prime \prime}$, respectively. As shown in Fig. 5,

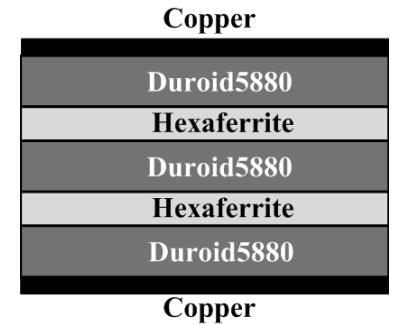

Fig. 4. The structure of a multi-layer substrate.
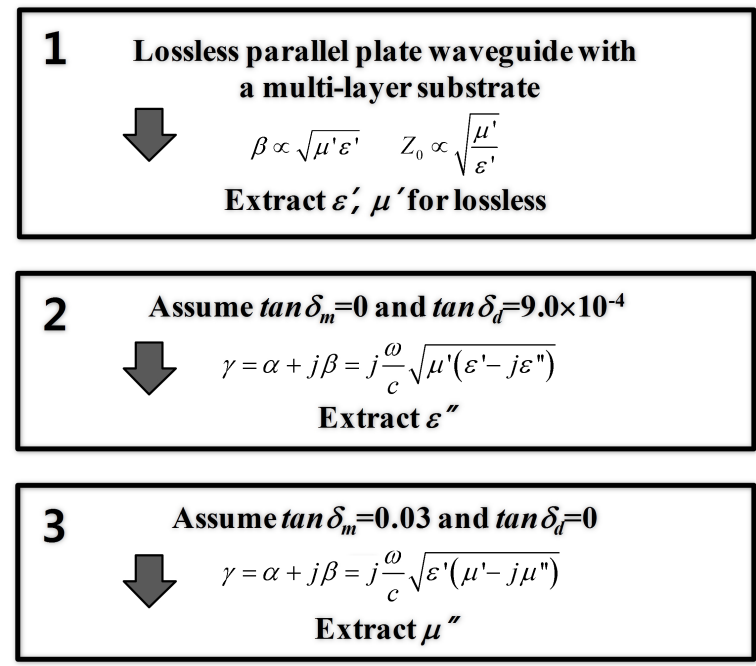

Fig. 5. Procedure to extract the equivalent parameters.

the equivalent parameters can be extracted in a few steps. First, $\varepsilon^{\prime}$ and $\mu^{\prime}$ can be extracted from the full wave simulated results for the parallel plate waveguide with a multi-layered substrate, assuming that $\varepsilon "$ and $\mu^{\prime \prime}$ are zero. To extract $\varepsilon^{\prime}$ and $\mu^{\prime}$, the simulated propagation constant of $\beta$ and the simulated characteristic impedance of $Z_{0}$ are compared with the analytical ones. Second, if only the dielectric loss tangent of the RT/duroid5880 is considered $\left(\tan \delta_{d}=9.0 \times 10^{-4}\right)$ and the hexaferrite is considered as lossless, a complex propagation constant of $\gamma$. is can be expressed as step 2 in Fig. 5 and $\varepsilon$ " can be calculated from the following equation:

$$
\varepsilon^{\prime \prime}=\frac{\alpha \beta c^{2}}{2 \mu^{\prime}(\pi f)^{2}} .
$$

Similarly, from step 3 in Fig. $5 \mu$ " can be written by:

$$
\mu^{\prime \prime}=\frac{\alpha \beta c^{2}}{2 \varepsilon^{\prime}(\pi f)^{2}}
$$

where a magnetic loss tangent $\left(\tan \delta_{m}=0.03\right)$ is considered. For the multi-layered substrate in Fig. 4, we can obtain $\varepsilon^{\prime}$ of $2.482, \tan \delta_{d}$ of $8.7354 \times 10^{-4}, \quad \mu^{\prime}=2.373$, and $\tan \delta_{m}$ of 0.0193 . The multi-layered substrate in 


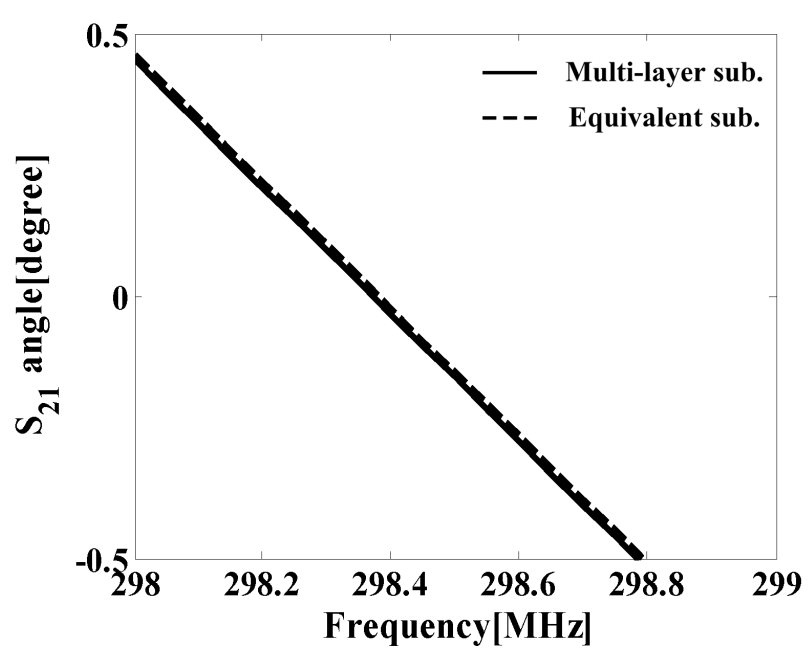

(a) Phase of $S_{21}$

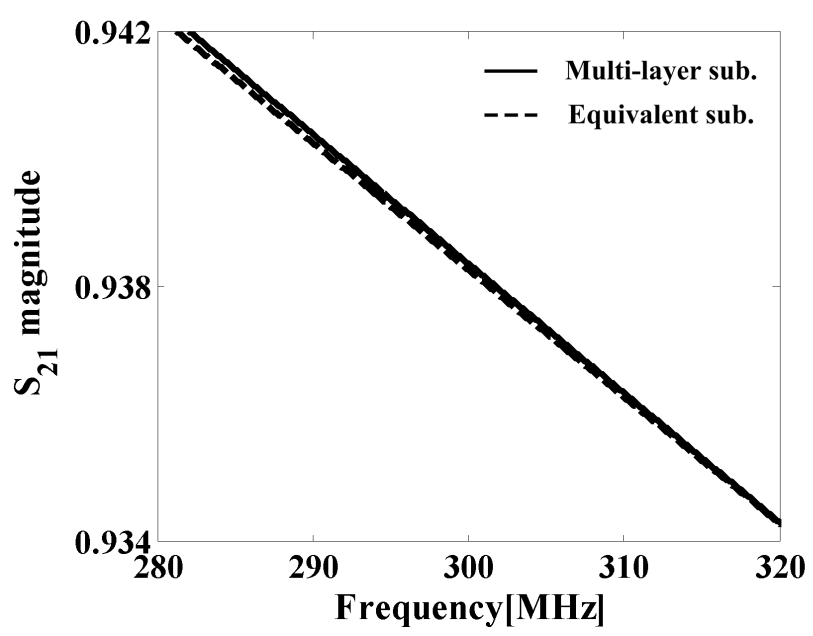

(b) Magnitude of $S_{21}$

Fig. 6. Simulated results.

Fig. 4 not only has a lower $\tan \delta_{m}$ but also has a smaller relative permeability when compared to a hexaferrite substrate.

We validated our extraction by comparing the phase and magnitude of the $S_{21}$ for a parallel plate waveguide having a multi-layered substrate with those of the $S_{21}$ for a parallel plate waveguide having an equivalent substrate $\left(\varepsilon^{\prime}=2.482, \tan \delta_{d}=8.7354 \times 10^{-4}, \mu^{\prime}=2.373\right.$, and $\tan \delta_{m}=$ 0.0193 ), as shown in Fig. 6. The results show an excellent agreement, indicating the validity of our extraction.

\section{Measurement}

Fig. 7 shows the one unit cell ENG ZOR patch antenna. The RT/duroid5880 substrates are located in the top and bottom layers and two layers of Co2Z-type hexaferrite are inserted between the RT/duroid5880 substrates. To obtain a lower material loss, air spaces of 5

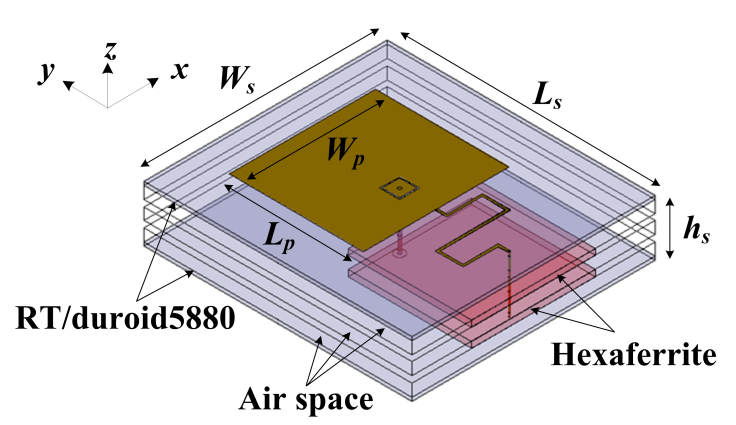

Fig. 7. Structure of a one unit cell ENG ZOR patch antenna $(k r=0.32)$.

$\mathrm{mm}$ are then added between each layer. In fact, the hexaferrite has a limited size $(50.8 \times 50.8 \times 2.5 \mathrm{~mm})$. The Co2Z-type hexaferrite layers are placed toward the via, as shown in Fig. 7, because the magnetic field of the antenna is concentrated at the via. In order to match 50 Ohm, a rectangular gap $(0.5 \mathrm{~mm})$ is inserted between the feed and the patch. The antenna $(k r=0.32)$ has the following dimensions: patch width $\left(W_{p}\right)$ of $60 \mathrm{~mm}$ $\left(0.040 \lambda_{0}\right)$, patch length $\left(L_{p}\right)$ of $55 \mathrm{~mm}\left(0.037 \lambda_{0}\right)$, substrate width $\left(W_{s}\right)$ of $100 \mathrm{~mm}\left(0.067 \lambda_{0}\right)$, substrate length $\left(L_{s}\right)$ of $110 \mathrm{~mm}\left(0.073 \lambda_{0}\right)$, and height $\left(h_{s}\right)$ of $23.14 \mathrm{~mm}\left(0.015 \lambda_{0}\right)$.

The measured $S_{11}$ is shown in Fig. 8. The solid line shows the simulated $S_{11}$ and the dashed line shows the measured $S_{11}$. In the simulated results, the antenna has an operating frequency of $199.2 \mathrm{MHz}$, a $10 \mathrm{~dB}$ bandwidth of $0.88 \%$, and a radiation efficiency of $5.3 \%$. The measured operating frequency is $196.1 \mathrm{MHz}$, the 10 $\mathrm{dB}$ fractional bandwidth is $0.99 \%$, and the radiation efficiency is $6.1 \%$. Thus, the measured results are in good agreements with the simulated results. This antenna has a poor radiation efficiency because of its material loss.

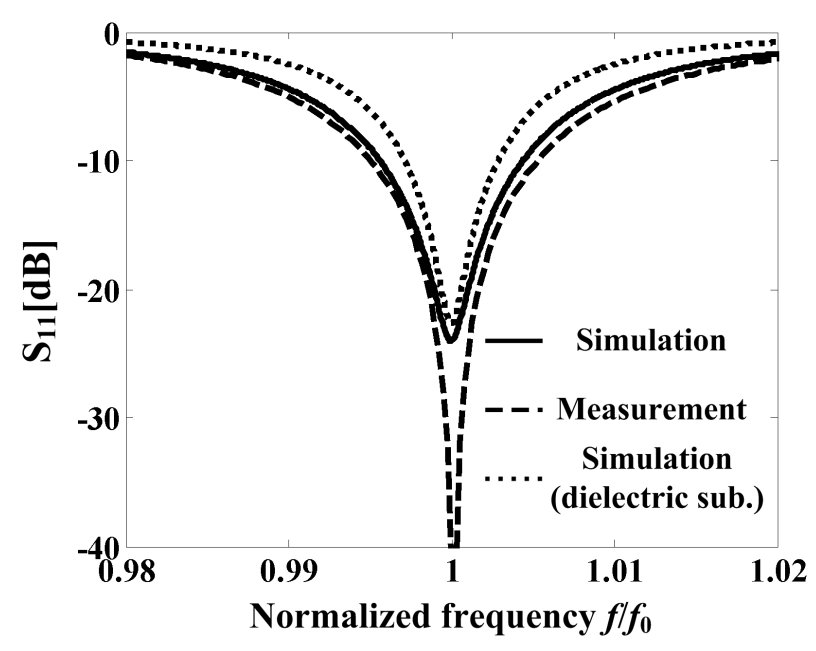

Fig. 8. Return losses. 


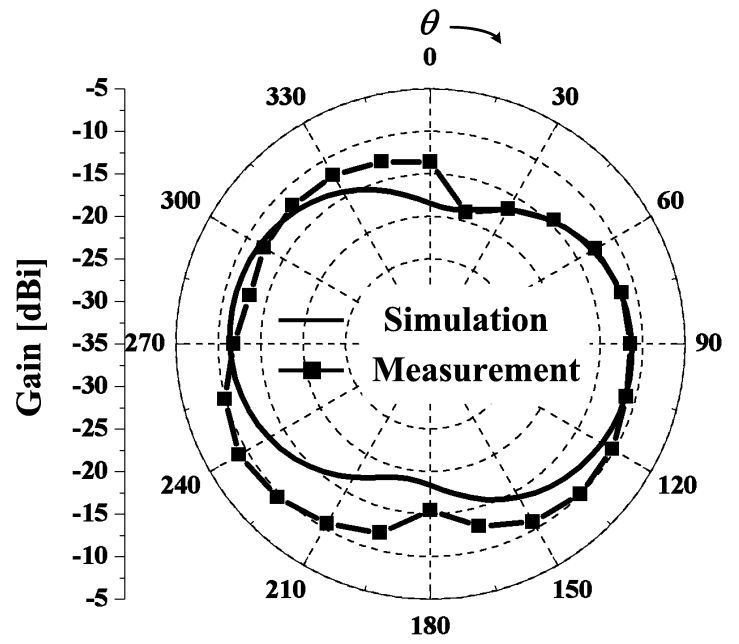

(a) E-plane pattern (y-z plane)

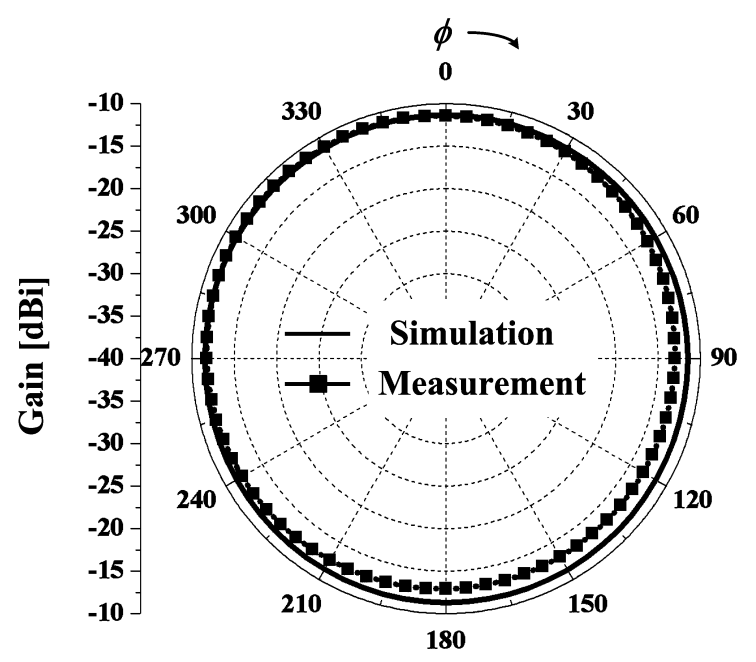

(b) H-plane pattern (x-y plane)

Fig. 9. The far-field radiation pattern.

However, if the $\tan \delta m$ of the hexaferrite substrate was $3.0 \times 10^{-4}$, the radiation efficiency of this antenna would be increased up to $32.5 \%$. A higher efficiency ENG ZOR antenna could also be obtained by increasing the thickness of the copper. For example, if the $\tan \delta_{m}$ of hexaferrite was $3.0 \times 10^{-4}$ and the thickness of copper was $0.17 \mathrm{~mm}$, the ENG ZOR antenna would have a radiation efficiency of $37.8 \%$ according to the simulated result. If this antenna is built on a pure dielectric substrate $\left(\varepsilon_{r}=2.5, \mu_{r}=1\right.$ and $\left.\tan \delta_{d}=6.0 \times 10^{-3}\right)$ instead of a multi-layered substrate, the operating frequency, the $10 \mathrm{~dB}$ fractional bandwidth, and radiation efficiency would be $198.7 \mathrm{MHz}, 0.57 \%$, and $5.2 \%$, respectively. Note that the dielectric constant $\left(\varepsilon_{r}=2.5\right)$ and the loss tangent $\left(\tan \delta_{d}=6.0 \times 10^{-3}\right)$ of the dielectric substrate are chosen for the antenna to have the same frequency and efficiency as the multi-layered antenna. Since the fabricated antenna has an asymmetric substrate, extraction of the equivalent parameters is difficult just using the proposed method. However, we can expect that the substrate has a relative permeability that is larger than 1 .

Finally, Fig. 9 shows the measured far-field radiation pattern. The fabricated antenna has an omni-directional pattern, as shown in Fig. 9(b). The simulated and measured peak gain are obtained as $-11.2 \mathrm{dBi}$ and -8.69 $\mathrm{dBi}$, respectively.

\section{Conclusion}

In this paper, a miniaturized ENG ZOR patch antenna $(k r=0.32)$ is proposed that has an improved bandwidth. The broad bandwidth is obtained for the ENG ZOR patch antenna by using a meandered via and a high permeability substrate. A multi-layered substrate is also designed to create the high permeability substrate. The ENG ZOR patch antenna using a meandered via and a high permeability substrate can have a broader bandwidth than a similar antenna using a straight via and a dielectric substrate. The $10 \mathrm{~dB}$ fractional bandwidth of the fabricated antenna is $0.99 \%$, which is 1.74 times as broad as that of the antenna with the dielectric substrate.

This work was supported by Mid-career Researcher Program through NRF grant funded by the MEST (No. 2010-0013273).

\section{References}

[1] J. H. Park, Y. H. Ryu, J. G. Lee, and J. H. Lee, "Epsilon negative zero ${ }^{\text {th }}$-order resonator antenna," IEEE Trans. Antennas Propag., vol. 55, no. 12, pp. 3710-3712, Dec. 2007.

[2] K. D. Jang, J. H. Kim, D. H. Lee, G. H. Kim, W. M. Seong, and W. S. Park, "A small CRLH-TL metamaterial antenna with a magneto-dielectric material," IEEE Antennas and Propagation Society International Symposium, Jul. 2008.

[3] S. T. Ko, B. C. Park, J. H. Park, and J. H. Lee, "Small epsilon negative ZOR antenna with improved bandwidth," The Journal of Korea Electromagnetic Engineering Society, vol. 19, no. 8, pp. 920-926, Apr. 2008.

[4] H. Mosallaei, K. Sarabandi, "Magneto-dielectrics in electromagnetics: concept and applications," IEEE Trans. Antennas Propag., vol. 52, no. 3, pp. 15581567, Jun. 2004.

[5] S. T. Ko, J. H. Lee, "Miniaturized ENG ZOR antenna with high permeability material," IEEE Antennas and Propagation Society International Symposium, 
Jul. 2010.

[6] J. K. Ji, G. H. Kim, and W. M. Seong, "Bandwidth enhancement of epsilon negative zero $^{\text {th }}$-order resonator antennas using a $\mathrm{Co}_{2} \mathrm{Y}$ hexagonal ferrite helical loading," Microwave Opt. Tech. Lett., vol. 53, no. 1, pp. 87-90, Jan. 2011.

[7] A. Sanada, C. Caloz, and T. Itoh, "Planar distributed structures with negative refractive index," IEEE Trans.

\section{Seung-Tae Ko}

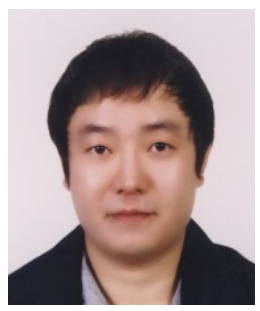

received the B.S. degree in electronic and electrical engineering and the M.S. degree in electronic information and communication engineering from Hongik University, Seoul, Korea, in 2006 and 2009, respectively, where he is currently working toward the Ph.D. degree. His research interests include metamaterial RF devices.
Microw. Theory Tech., vol. 52, no. 4, pp. 1252-1263, 2004.

[8] D. M. Pozar, Microwave Engineering, New York: Wiley, 1998.

[9] R. C. Hansen, M. Burke, "Antennas with magneto-dielectrics," Microwave Opt. Tech. Lett., vol. 26, no. 2, pp. 75-78, Jul. 2000.

[10] http://www.trans-techinc.com

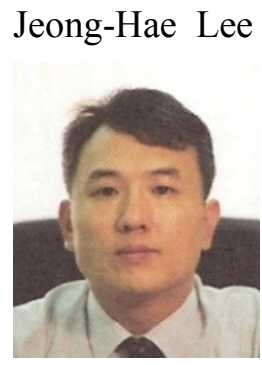

Scientist of General Atomics, San Diego, CA, where his major research was to develop the millimeter wave diagnostic system and to study the plasma wave propagation. Since 1996, he has been at Hongik University, Seoul, Korea, where he is a Professor of Department of Electronic and Electrical Engineering. His current research interests include the microwave/millimeter wave circuits, the millimeter wave diagnostic, and the metamaterial RF devices. 